\title{
WHO'S MISSING FROM THIS PICTURE? THE UNDERREPRESENTATION OF OLDER EMPLOYEES ON ORGANIZATIONAL CAREER WEBSITES AND ITS CONSEQUENCES
}

\author{
Lillian Little, Salem State University \\ Scott DeWolfe, Salem State University
}

dx.doi.org/10.18374/JIMS-13-4.6

\begin{abstract}
The percentage of older individuals in both the population and in the labor force is growing at an unprecedented rate that outpaces that of younger age groups. Like the rest of the job-seeking population, a great many of those older individuals are utilizing organizational websites to start the job search process and are likely to perceive messages from images, in addition to text, on those websites. The present study was designed to build a case using previous research to illustrate the negative impact if older individuals are underrepresented on the careers link of organizational websites, and to determine whether such underrepresentation is occurring. Results of preliminary descriptive statistics suggest that underrepresentation of images of older individuals is occurring on organizational websites, and previous research suggests that it can result in a negative impact for older job-seekers. Due to dramatic changes in demographics, the number of older Americans seeking employment has risen to record levels in recent years. Simultaneously, the use of organizational career websites has become a first step in the job search process for many older people. If, as a result of the content of career websites, older job seekers are less likely to apply for jobs, a significant number of them are likely to be adversely impacted. The goals of the present study are to examine the process by which a lack of images of older workers on organizational career websites can influence perceptions of older job seekers and to determine whether organizational websites do, in fact, underrepresent images of older workers. By empirically determining whether older employees are underrepresented on organizational websites and by presenting arguments drawn from several disciplines as to why such underrepresentation may lead to discouragement in seeking employment, the present study aims to achieve those goals.
\end{abstract}

\section{Keywords:}

